Cest te Journal of Engineering Science and Technology Review 8 (3)

Research Article
JOURNAL OF

Engineering Science and

Technology Review

\title{
Standardization of the Experimental Methodology for Quality Assurance and Quality Control (QA-QC) of the CLIC Structural Materials
}

\author{
N. Gazis $* 1,2$, S. Stapnes ${ }^{2,3}$ \\ 1 \\ ${ }^{1}$ National Technical University of Athens, Athens, Greece \\ ${ }^{2} \mathrm{UiO}$, Oslo, Norway \\ ${ }^{3}$ CERN, Geneva, Switzerland
}

Received 25 March 2015; Accepted 26 April 2015

\begin{abstract}
The main linear accelerators (linacs) of the Compact LInear Collider (CLIC) are constituted of sequential two-beam modules (of approximate length of two meters). The CLIC linacs need to be firmly stabilized on their supports with a micron-level requirement, essential for maintaining the final target luminosity close to the required XXX value. Real scale two-beam prototype modules have been designed, manufactured and commissioned to study their behaviour under different operation modes and experimental conditions.

The CLIC machine will work for continuous runs under conditions of high radiation background. The structural materials of the systems of the accelerator have to sustain the significant fatigue and activation due to the radiation, generated mainly by the losses of the particle beam. Extensive testing has taken place with a combination of mechanical experiments and irradiation sessions on samples of structural materials, focusing on the micro-precise CLIC module supporting system. The followed experimentally strategy was standardized in a series of sequential steps.
\end{abstract}

Keywords: Quality Assurance, Quality Control, Clic,

\section{Introduction}

In this work, a novel standard quality assurance and quality control (QA-QC) methodology is proposed allowing for the efficient validation of specimens from a pool of potential future CLIC materials. The results of the different types of tests can be propagated back to the material selection of each subsystem, affecting also the corresponding engineering design. Although the present work was performed within the context of CLIC engineering design and focuses on the CLIC module supporting system, it is also applicable to the design and manufacturing of other future accelerator systems as well and therefore it may consolidate a standard quality assurance and quality control methodology for the selection of accelerators structural materials.

\section{CLIC layout and components}

The CLIC study (Figure 1) is focused [1] on the design of an e- e+ linear collider, where an electron beam collides with a positron beam at the interaction point (IP). The maximum colliding beam energy at $3 \mathrm{TeV}$ will have a total luminosity of $2 \cdot 10^{34} \mathrm{~cm}^{-2} \mathrm{~s}^{-1}$.

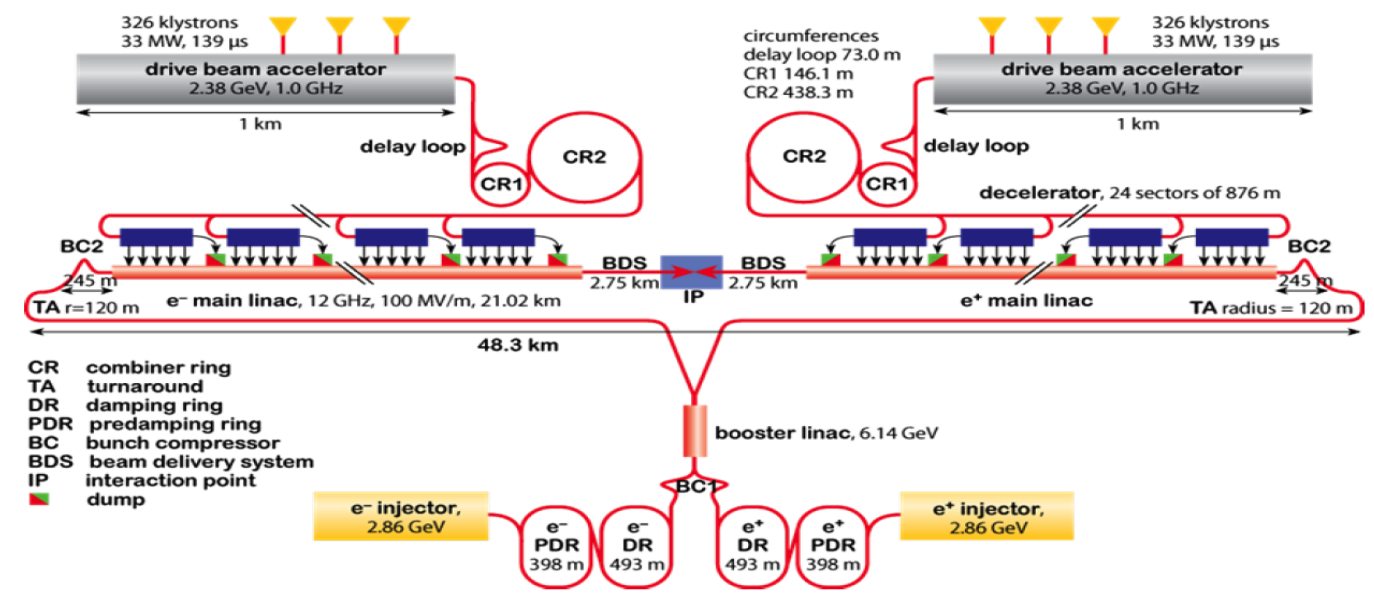

Fig. 1. The CLIC layout at $3 \mathrm{TeV}$ colliding beam energy

\footnotetext{
* E-mail address: Nick.Gazis@cern.ch

ISSN: 1791-2377 @ 2015 Kavala Institute of Technology. 
The CLIC two-beam configuration is formed by repeated "modules". The modules (or two-beam modules) are the smallest repetitive units of the collider assembled in series to form both linacs, with their parallel beams, the Drive Beam (DB), where the power is generated and extracted and the Main Beam (MB) in which the particle beam is accelerated [2] to the final beam energy. The modules include all the main RF components [3] and the focusing magnets of the machine.

The challenging optics (beam focusing) requirements for the particle beam define several different types of modules with MB-quadrupole magnets of different lengths [3]. The
MB of the module Type- 0 contains only Accelerating Structures whereas the modules of Type- 1 up to Type-4 include also MB-quadrupoles of variable length. The MBquadrupoles take the place of two (2), four (4), six (6) or eight (8) Accelerating Structures (AS), respectively [3].

The two-beam module design has to take into consideration the engineering requirements for the different technical systems. The main components are designed to optimize the filling factor and gain in volume compactness of the CLIC accelerator. A typical two-beam module [4] is illustrated in Figure 2.

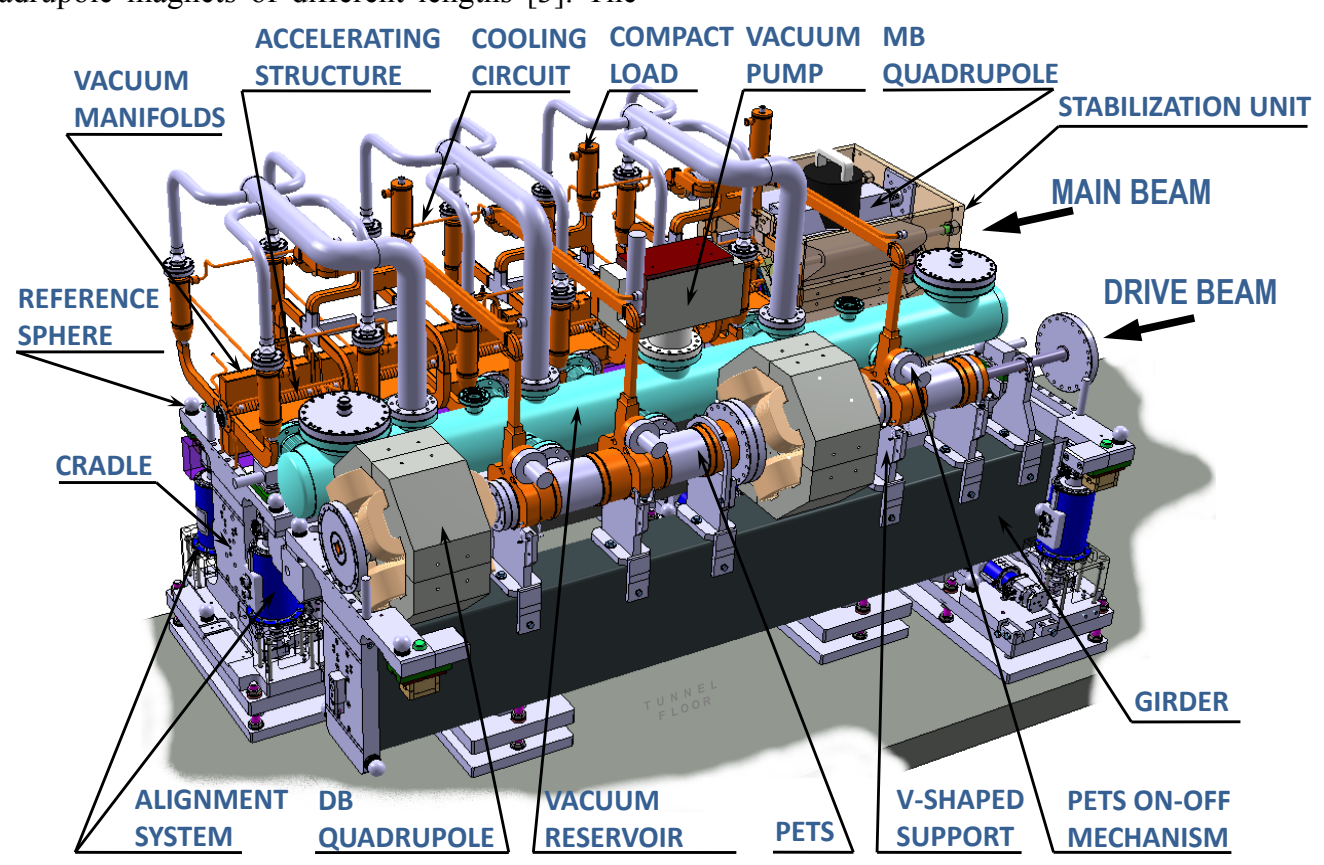

Fig 2. The CLIC two-beam module (Type 1)

All the RF components (with the exception of the MBquadrupoles) of the two-beam modules are installed and aligned on an innovative supporting system [5]. The module length is presently $2010 \mathrm{~mm}$ dictated by the mechanical and thermal stability of the overall system, along with several other technical parameters. The length of $30 \mathrm{~mm}$ is reserved for the inter-girder connection, which is referred to as Articulation Point [6]. The module supporting system is mechanically articulated throughout the entire length of the DB linac. The mechanical continuity of the support of the $\mathrm{MB}$ is interrupted only by the individual supports of the MB-quadrupoles. The supporting system is constituted out of several components (girders, V-shaped supports, cradles, U-clamps, etc.) [7] to fulfil the various technical requirements for support and stabilization of the RF components. The in-depth investigation and understanding of the behaviour of the supporting elements is essential for the successful alignment and operation of the modules.

\section{Criteria for the selection of the testing materials}

The supporting system has played the role of model system for the determination of the experimental methodology for the selection of the module structural materials. Its proximity to the particle beam, thus (i) the high radiation background in which it is operating, in combination with (ii) the imposed high precision tolerances on its design (both geometrical and positioning) make it an excellent example of the system which needs to be tested for assessing its mechanical properties. In addition, (iii) the diversity of the potential structural material, provide an experimental comparison and reference for several categories of materials, such as ceramics, composites and metals.

(iv) The structural materials of the CLIC supporting system need to satisfy several mechanical and radiation hardness requirements. The radiation hardness properties of the materials may have a significant influence on the material ageing effects and consequently on the safety and environmental impact of the facility. Moreover, (v) potential radio-activation of the CLIC materials could result in radiation doses emitted to the environment and technical personnel, which could complicate the handling and maintenance of the CLIC components. In addition, (vi) longterm radio-activation of the CLIC materials could result in products, which require special treatment, storage or disposal as radioactive waste at the end of the life of the accelerator facility.

Also, (vii) the fatigue of the materials due to the high radiation background could inflict critical issues according to each case. (viii) Such issues could result in alteration of the material mechanical properties, shortening of the life cycles of the components or even alteration of the material microstructure.

Finally, (ix) the dynamic loads with their corresponding residual stresses, applied on the materials in combination with high radiation background, could inflict rapture in a macroscopic level. Such events would have a negative impact for the CLIC supporting system and thus it was essential to proceed to combined irradiation and mechanical testing. 
Radiation testing

The CLIC machine will operate under severe radiation background level [8] for a long time period. The supporting system is situated in a zone of a high radiation dose [9], closely beneath the RF components. The radiation background is mainly the results of the function of the RF components i.e. by the particle beam acceleration (AS) and deceleration (PETS). The investigation of the resulted ageing effects on the technical system materials and the designed experiments are based on the latest available radiation simulations for the CLIC beam losses [10].

Based on the existing simulation $[8,10]$ of the CLIC nominal operation; photons $(\gamma)$, electrons $(\mathrm{e}-)$, positrons $\left(\mathrm{e}^{+}\right)$ and neutrons (n0) are mainly dominant in the low energy range and up to a maximum energy of $1 \mathrm{GeV}$ for the majority of them. The protons $\left(p^{+}\right)$, pi-plus $(\pi+)$, pi-minus $(\pi-)$ and muons $(\mu \pm)$ are appearing approximately in the medium energy range of $10 \mathrm{MeV}$ with a maximum energy going up to $10 \mathrm{GeV}$. A special focus is needed at the energy range of the two-beam module area (with respect to the rest of the CLIC tunnel cross section), where the radiation background is mostly intensive by almost a gain factor of 102-103.

The irradiation experiments were performed within the context of evaluating the activation properties of the two candidate structural materials of the supporting systems of the CLIC module: sintered silicon carbide (SiC) and Epument 145/B.

The sintered $\mathrm{SiC}$ combines the material physical properties of high hardness, low density, low porosity; good wear resistance, excellent corrosion resistance, low thermal expansion and high thermal conductivity leading to excellent thermal shock resistance.

The Epument $145 / \mathrm{B}$ is a three-component mineral cast polymer based on an epoxy resin including a special filler combination consisting of raw materials. The Epument material offers characteristics like highest rigidity, low thermal conductivity, thermal expansion coefficient adjusted to structural steels, lowest creep behaviour under stress influence and relatively low cost for its applications.

A detailed evaluation of the activation properties of a material requires knowledge of the following parameters:

- Module geometry,

- Materials composition, even in impurity levels,

- Radiation background (type, energy spectra, fluxes),

- Nuclear reaction cross-section with each of the sample contained elements in reference to the energy of interest,

- Accelerator machine operation time scheme.

The neutron activation properties of the structural materials (like e.g. SiC and Epument) have been examined by neutron irradiations in the facility of the Tandem Van der Graff Accelerator of the NCSR "Demokritos", Athens, Greece. The compositions of the material samples were determined at a preliminary level, and the induced activation for neutron irradiation up to $50 \mathrm{MeV}$ was evaluated. According to the existing simulations of CLIC radiation doses, the irradiation neutron field reflected roughly $60 \%$ of the neutron flux for the CLIC nominal operation scheme; which is considered as a very satisfactory level for the current study.
The neutron fluence and energy spectrum of the Tandem Accelerator was characterized by the Multiple Foils activation technique [11].

The composition of ceramic materials and metals was obtained from the data provided by the manufacturer and the bibliography. However, the material composition was crosschecked by measurements performed using the X-Ray Fluorescence (XRF) technique. For the composite materials such as the Epument 145/B (typical three-component mineral cast polymer based on an industrial epoxy resin), the XRF measurements are expected to reveal a large variation of uncertainty in elemental composition due to matrix heterogeneity. Clearly, the XRF is not the method of choice for the elemental analysis of such inhomogeneous materials; so other techniques are recommended, i.e. the Neutron Activation Analysis (NAA). Nevertheless, the XRF is an excellent method for the comparative study of the composition of the before mentioned categories of materials (e.g. ceramics, fine ceramics, composites), which could provide additionally a "macroscopic" average composition.

Radiation activity calculations of several experimental energy frontiers (of neutrons) were performed as a function of time after the end of irradiation sessions. It is significant to note that the assumed CLIC accelerator operation scheme comprises 7.5 irradiation cycles of 180 days beam-on and 185 days of maintenance (beam-off). [3] After the end of the final experimental cycles, activity calculations need to be performed at the end of the irradiations and at discriminated post-irradiation time periods [12]. For the neutron irradiation energy spectrum values, the specific activity was calculated at the end of each irradiation cycle. The predominant nuclides were assessed with respect to the total activity. In the subsequent time period of days for the irradiations, the post-irradiation activity needs evaluation with respect to time and to the determination of the predominant nuclide, as well as estimation of the resulting dose-rate.

In Figure 3, the evaluated specific activity for SiC samples irradiated by $1 \mathrm{MeV}, 20 \mathrm{MeV}$ and $50 \mathrm{MeV}$ neutrons is shown as a function of post-irradiation time. The dominant nuclides in these neutron energies and time cycles were ${ }^{55} \mathrm{Fe}$ and ${ }^{58} \mathrm{Co}$. It is noted that irradiation with higher energy neutrons resulted in a lower activity for the ceramics [11]. Thus, the specific activity has to be documented along with the dominant nuclides, with respect to the total activity. For the post-irradiation days, the total activity has to be measured also with respect to the predominant nuclides and their respective contributions to the total activity. At this step, it is expected that the dominant nuclides can differ in between the post-irradiation measurements.

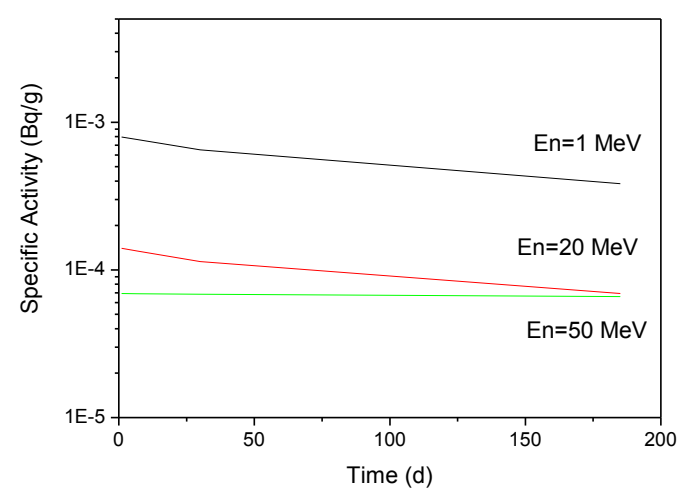

Fig. 3. $\mathrm{SiC}$ specific activity as a function of time post-irradiation for samples irradiated at 1,20 and $50 \mathrm{MeV}$ neutrons 


\section{Mechanical testing}

The behaviour and corresponding reaction of the materials under external strain-causing factors is controlled via their mechanical properties. The resistance that each material demonstrates towards the imposed loading conditions constitutes the interaction way that pertains the structural behaviour for each component. Therefore, the operational conditions are simulated by the distresses (static or dynamic) of the testing. The testing analysis and result reproduction in situ provides a characterization of the expected behaviour for each part.

In order to proceed to such a material characterization, as previously described, the strain-stresses curves were experimentally produced [13]. Fundamental sizes of the tested materials were included in these curves such as the modulus of elasticity, the plastic behaviour, the limit of fracture stress, etc.

The testing methodology that was utilized corresponded to norms for brittle materials; since all tested materials are categorized as ceramics, fine ceramics or composites. In addition, experimental results for the metal materials were retrieved from the existing literature. The experimental findings for the ceramics and composites were then compared to the literature results for metals and verified. [14]

The temperature of the testing, matching the operational conditions, was categorized as low temperature: [15]

$T_{\text {oper }}=T_{\text {test }}<10 \times T_{\text {melt }}$

Where the corresponding variables stand for $T_{\text {oper }}$ : temperature of operational conditions, $T_{\text {test }}$ : temperature of the testing conditions, $T_{\text {melt }}$ : temperature of the melting point of the material.

At this point it is worth mentioning that in the brittle fracture of the strength testing we came across with both possibilities of:

- Transgranular brittle fracture

- Intergranular brittle fracture

The reason was that each of the tested materials, likely, produced different cases of fracture, giving us the possibility to compare the results. The micro-mechanism of the fracture of a material could differ according to the:

- Testing setup

- Loading methodology

-Size effect ${ }^{*}$

For this reason, the followed experimental methodology is proposed as a part of the standardization methodology of this work, so as to avoid potential incomparable results coming out of the several testing cases.

The mechanical testing was initialized with the mechanical strength tests. For this phase, the simple Uniaxial Compression Test $\left(U C T^{\dagger}\right)$ was chosen as a testing procedure. It is worth mentioning that the tensile strength testing is the most common (and usually first applied)

* Size effect: The dependence of the measured mechanical properties on the size of the (self-similar) specimens used for the laboratory tests.

${ }^{\dagger}$ In the current citation the UCT is always unconfined. mechanical test for pilot cases. However, one additional reason for such a testing choice was that all supporting system components were subsequent to real-time compressive loads during their operation. The common results extracted from the simple compression (and potentially the drawing/tensile testing) were:

-Experimental data of both tests with similar strain-stress graphs for a dedicated material

- Similar or identical loading values applied for the compressive/drawing testing conditions

The main qualitative differences in between the before mentioned UCT tests versus tensile strength testing are:

-The directions for the application of the testing loads are opposite for each of these two different mechanical tests.

- The result of the simple compression testing is the increase of the transversal direction of the specimen, whereas during the drawing testing the longitudinal dimension of the sample is being increased.

The simple compression methodology is flexibly applicable to the use of either cylindrical or cubical samples for the experiments. The standardized cubical samples were chosen according to the easiness of their fabrication.

\section{Mechanical testing of structural materials for the validation of strength}

For several categories of brittle materials such as ceramics, composites but also earthen and porous materials (such as Epument 145/B) the modulus of elasticity under compression is measured to be higher than the modulus of elasticity under tensile stress. However, for the ceramics (such as $\mathrm{SiC}$ ) such a fact is limited, corroborating the equal values of the experimentally acquired modulus of elasticity by compression and tensile tests respectively. In addition, the known fact from the literature was that all tested similar materials were subsequent to Hooke's law only for an extremely limited region (if it existed at all) of their (tested and measured) mechanical behaviour. $[11,16]$

It is underlined that the yield point and the fracture point have almost an identical value for this type of material. In similar cases, these two mechanical properties are considered to have almost identical values, when referring to brittle materials.

The quantities recorded during the tests were the time of the experiment, the load and the axial displacement. The raw data were properly processed with the aid of commercial software [Mathcad, Microsoft Excel ${ }^{\mathrm{TM}}$ ] to eliminate the experimental noise. The stresses were calculated afterwards with the help of a dedicated computational algorithm. The axial stress-strain diagrams were obtained from the elaborated data. Typical examples of such curves are plotted in Figure 4.

From the curves of Figure 4, it became possible to calculate the values of some characteristic mechanical quantities such as $\sigma_{\text {peak }}$ : peak stress, $E$ : Modulus of elasticity and the $W$ : strain energy density spent up to the peak load or up to the first visible external crack. The following formula was used to calculate $W$. Numerical integration was not considered since there was no evidence of external crack appearing before the peak stress:

$W=\frac{\sigma_{\text {peak }} \times \varepsilon_{\text {peak }}}{2}$ 


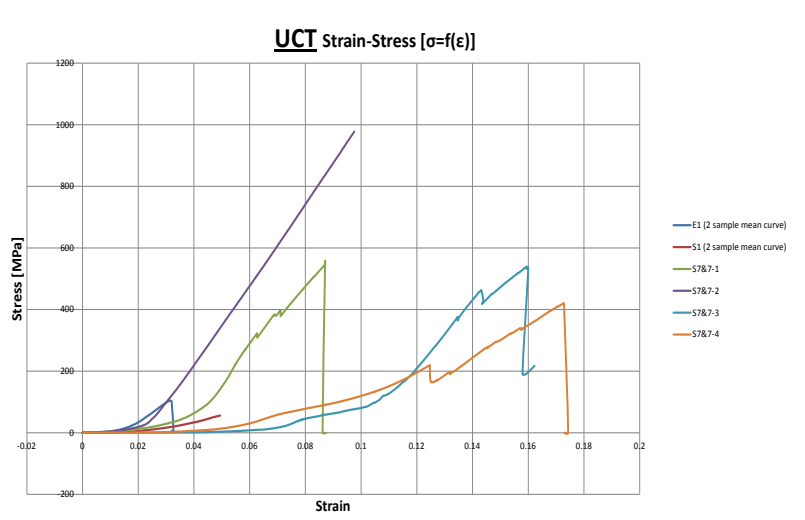

Fig. 4. Summarizing UCT stress-strain graphs

The stiffness of the materials of the specimens was the main reason for the observed behaviour: The average values of the peak stress and of the rest of the before mentioned measured properties were then summarized [5], [11]. The peak stress evidently presented a strong dependence to the sample dimensions.

In several times, it appeared that specimens behaved as a structure rather than as a homogenous material. Regarding the modulus of elasticity of the specimens, it appears that its value also depends on the size of the samples, contrary to what is perhaps expected. Again this observation is in good qualitative accordance with the respective literature [14], [16].

The working load of the tested materials, in practice, is restricted to a portion only of the $\sigma-\varepsilon$ graph, far below the peak stress. The values of the modulus of elasticity varied between extended limits, while the respective scattering was noticeable. The before mentioned observation could be explained by taking into account that the energy density includes the effect of deformations in all three directions. The influence of the specimen size, affects the tendency of the material to dilate and expand perpendicularly to the loading direction. By this way, its failure mode is determined, proving by itself the variation of the mechanical strength data [11].

The irradiated specimens prove to have most of their properties $(E, v)$ similar to the reference. The noticeable difference exists on the maximum limit of the measured strains. It appears that the radiation provokes a bigger deformation potential for the tested materials. Such a result could be translated as reduced E (which is evident) and additionally reduced stiffness in terms of material microstructure. Based on the known literature [5]. [11]. [12] regarding the behaviour of the brittle materials under high radiation, we can vouch that such results were expected and confirmed.

The mechanical testing continued with the three-point bending $(3 P B)$ experimental procedure. Three-point bending is considered, by the engineering community, as one of the most popular mechanical tests and is widely used both for practical reasons as well as for purely scientific applications. $[17,18]$ Again at this point, the boundary conditions of the module components could be modelled according to the three-point bending. So, with the $3 \mathrm{~PB}$, the fundamental part of the module supporting system was modelled and tested under simulated conditions matching its operation.
The main conclusions of the experimental study for $\mathrm{SiC}$ and Epument $145 / \mathrm{B}$ can be summarized among the following points:

-The failure load varied between the limits of $2000 \mathrm{MN}<$ $\mathrm{P}_{\mathrm{F}}<5000 \mathrm{MN}$.

-The fracture started always from the lower fibre and propagated perpendicularly to the axis of the specimen towards the loading point.

- The influence of the concentrated load is significant in a small area around the point of application, distributing the strain field only in the section exactly below it. Such influence becomes negligible at a distance " $z$ " smaller than the half height of the specimen.

\section{Results and discussion}

The current work aims at documenting the strategy methodology which can be used to test, validate and verify the structural materials for the CLIC machine. The quality assurance and quality control (QA-QC) of the accelerator components is dependent on the standardized testing strategy of the accelerator materials. Also, the structural materials of the parts of particle accelerators of similar class should undergo such QA-QC testing. Major influence on the study and standardization are of course the radiation levels and the type of mechanical fatigue that is imposed on the systems of each accelerator. The testing results along with the analytical simulation data were part of an extended study, which is summarized in a PhD thesis [11].

The tested materials have been approved and published at the CLIC CDR [3], after a long optimization process influenced also by the cutting edge manufacturing techniques.

The results of the present study showed that a careful investigation of the radiological properties for the CLIC structural materials is required for occupational radiation protection and waste management for the components of such an accelerator. The results derived from the irradiation testing depend on the dedicated experimental conditions (along with their limitations) in combination to the calculation assumptions for the data analysis, which were identified and therefore cannot be readily generalized. The implementation of the neutron beam irradiation to the candidate materials of the CLIC module supporting system at different neutron beam energies has been proved a very useful tool for the material study and analysis. The resulting investigation provided [11]:

- The sample composition, a necessary requirement for a detailed elemental analysis of the specific test specimens (i.e. NAA or XRF), which confirms and/or corrects experimentally the material composition provided by the supplier.

-The activation calculations, which give all the potential types of activating nuclides and their corresponding energies.

-The preparatory phase of the specimens for the following mechanical testing and analyses, which can provide all the structural information of the materials via the standard mechanical tests (UCT and 3PB).

As mentioned in the literature [13], [16], [18] and proven partially in the experimental results, the absolute value of the yield strength from the compression testing of each tested 
material is submultiple to the drawing yield (tensile) strength (according to the provided industrial datasheets). Moreover, the Epument 145/B cracking and failure, which appeared in UCT, is justified to have the shape of the shear fracture under an angle of approximately $45^{\circ}$ with respect to the axis of the loading condition. This comes from the theory that the brittle materials present low resistance to the shear stresses comparing to the normal compression stresses. The normal stresses in this case have the tendency to "close" the existing micro-cracks and anomaly regions of the internal material microstructure. So, the fracture commences in a higher point of loading under the normal stresses. The compressive fracture of the material is influenced by the internal structure and texture of the material. The boundary surfaces that exist in the microstructure of such a dedicated material result in a less resistant behaviour under compression. Consequently, the dedicated internal texture defines the "way" that the crack and fracture boundaries would follow with respect to the axis of the loading condition.

However, the flow/slide levels that appear in the compression tests verify that due to the experimental practical defaults, the absolute (ideal) uniaxial loading of the specimen is never applicable. In reality, the friction forces that rise in between the sample interfaces and the machine grips (with rigid end platens) of the testing machine create a triaxial force. For this reason, the actual dimensions of the tested specimen influence the results (the already mentioned size effect). The size effect dependency was expected to be acknowledged in the results, as it is common rule to appear to samples with dimensions smaller than $50 \mathrm{~cm}$, as in all of our experimental cases. The standardized methodology and specification that were followed for all the experiments reassured the objectivity and comparability of the results.

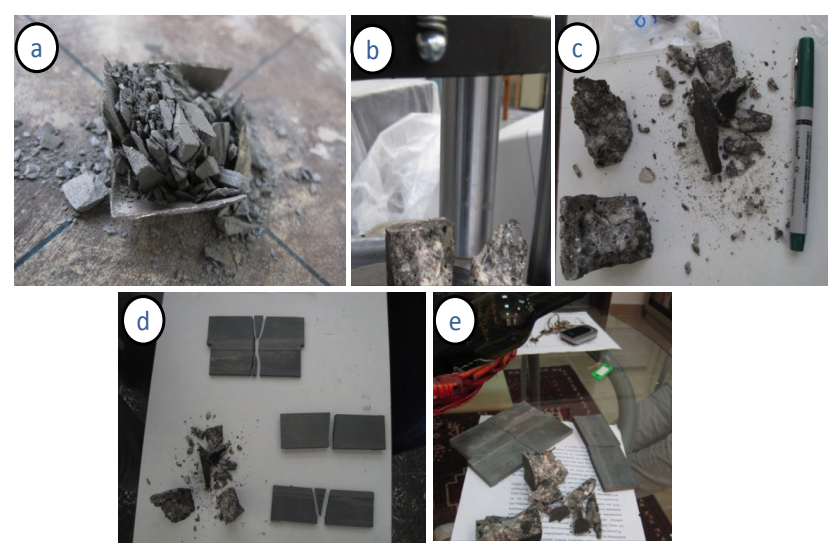

Fig. 5. Specimens after testing a) UCT specimen of $\mathrm{SiC}, \mathrm{b}) \mathrm{UCT}$ specimen of Epument 145/B, c) UCT specimen of Epument 145/B, d, e) 3PB specimens of Sic and Epument 145/B

The influence of the concentrated load during the threepoint bending is dominant on the failure mechanism of the macrostructure of the specimens, as illustrated in Figure 5. Although the issue of the often rough existing surface of the specimens was overpassed with the $\mathrm{Pb}$ shims, the fracture into more than two (2) pieces, confirms the influence of the surface anomalies on the failure mechanism and results. Also for this mechanical testing, the size effect played a significant role on the measured properties, without misquoting though their comparability.

It is also important to remark that the measured strains appear large with respect to the known hardness for such a material. It is also known that the deformations coming from the bending mechanical tests (e.g. 3PB) result in bigger values than the ones appearing on tensile mechanical tests. For this reason but also due to the reasonable order of magnitude of the absolute deformations (strains) we can testify their accuracy.

\section{Conclusions}

The extended study (UCT, 3PB, etc.) of the materials used for the prototype fabrications are presented under the mechanical testing of reference and irradiated specimens. The achieved neutron irradiations approached in a satisfying level $(70 \%-80 \%)$ the simulated radiation background of the future CLIC operation [7], [11]. The results from the neutron activation analysis were also extracted. A novel testing methodology was developed and standardized for the selection of the structural materials for the CLIC accelerator. The candidate materials should meet all the mechanical specification, essential for the successful operation of the module. In addition, material samples should pass through the novel experimental procedure:

- Irradiation under a high energy and fluence neutron beam,

- Mechanical testing before and after the irradiation,

- Quality and microstructure controls before and after the irradiation and the mechanical tests.

The last part of the experimental methodology standardization is the analysis of the data coming from the post-production experimental testing. The individual material radio-activation properties were studied. Following step are the irradiation sessions that fatigued in micrometric scale the materials. Finally the mechanical testing that followed, confirmed that the chosen materials could withstand both radiation and operation time with a very satisfying safety factor $(>3)$. Moreover, the experimental characterization of the materials by these means proved that the preliminary simulations, basis of the study, are successful and up-to-date since it is instated on a carefully planned engineering approach.

In parallel, data analyses of the mechanical tests of irradiated and reference specimens were achieved. The very first results are indeed very positive by revealing that no significant changes occur to the mechanical properties of the fatigued materials. The standardization of an experimental methodology is indeed a challenging task. However, the quality assurance and reliable results that such a standardized methodology can produce, are of prime importance towards the realization of a post-LHC era collider. 


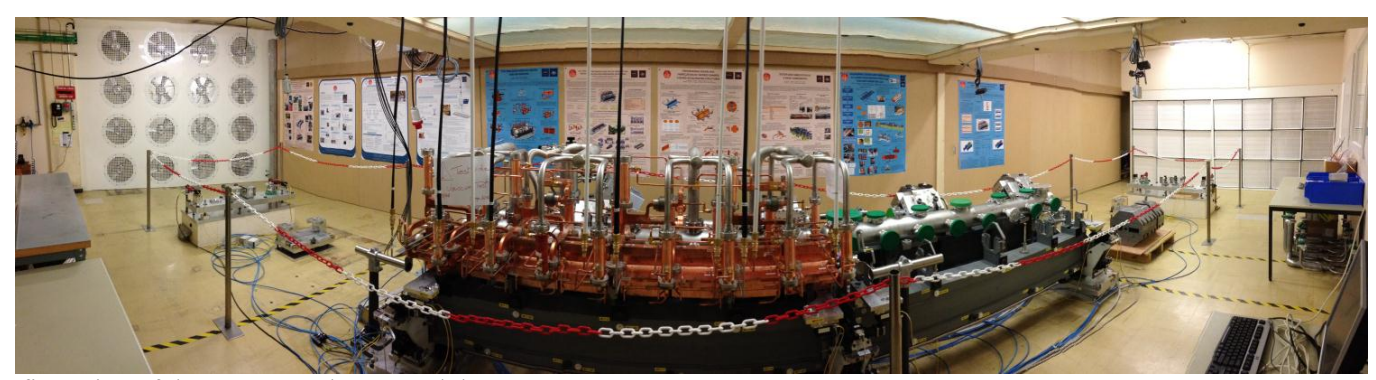

Fig. 6 Lab configuration of the CLIC two-beam module

\section{References}

[1] The CLIC website http://clic-study.org

[2] The CLIC Programme: Towards a staged $\mathrm{e}^{+} \mathrm{e}^{-}$:Linear Collider exploring the Terascale [CLIC Conceptual Design Report], CERN2012-005, Geneva, Switzerland, August 2012

[3] The CLIC Conceptual Design Report http://project-cliccdr.web.cern.ch/project-clic-cdr/, May 2012

[4] CLIC 2008 Parameters, F. Tecker et al., CERN-OPEN-2008-021, CLIC-Note-764, Geneva, Switzerland, June 2008

[5] Study of the supporting system for the CLIC Two-Beam Module, N. Gazis, G. Riddone, H. Mainaud-Durand, A. Samoshkin, D. Gudkov, S. Simopoulos, E. Hinis, T. Alexopoulos CLIC-Note857), pp. 201-206, Issue 2, Vol. 4, Journal of Engineering Science and Technology Review [ISSN:1791-2377], September 2010

[6] Study and application of micrometric alignment on the prototype girders of the CLIC Two-Beam Module, N. Gazis, M. Anastasopoulos, G. Riddone, A. Samochkine, H. Mainaud-Durand (CLIC-Note-878), pp. 96-99, Vol. 495, Key Engineering Materials [ISBN-13:978-3-03785-292-7], Trans Tech Publications, Switzerland, November 2011

[7] Fabrication and validation of the prototype supporting system for the CLIC Two-Beam Modules, N. Gazis, G. Riddone, S. Griffet, A. Samoshkin (CERN-ATS-2011-047), pp. 1015-1017, TUPC012, 2nd International Particle Accelerator Conference 2011, San Sebastian, Spain, September 2011

[8] Requirements of a beam loss monitoring system for the CLIC TwoBeam Modules, S. Mallows at al. (CERN-ATS-2011-066) pp. 2385-2387, WEPC171, 2nd International Particle Accelerator Conference 2011, San Sebastian, Spain, September 2011

[9] The CLIC machine protection, M. Jonker et al., pp.2860-2862, WEPEB071, 1st International Particle Accelerator Conference, Kyoto, Japan, May 2010

[10] Requirements of CLIC Beam Loss Monitoring System, M. Sapinski, et al. (CERN-ATS-2010-105), pp.2869-2871, WEPEB074, 1st International Particle Accelerator Conference, Kyoto, Japan, May 2010

[11] Development of the Supporting System for the CLIC Two-Beam Module, N. Gazis, PhD thesis submitted to the School of Engineering at the National Technical University of Athens

[12] The neutron facility at NCSR "Demokritos" - Implementation in the case of $232 \mathrm{Th}(\mathrm{n}, 2 \mathrm{n})$ reaction, R. Vlastou, C.T. Papadopoulos, G. Perdikakis, M. Kokkoris, C.A. Kalfas, S. Kossionides, D. Karamanis and P.A. Assimakopoulos, pp. 324-328, Vol. 701(1), American Institute of Physics Conference proceedings, 2004

[13] A treatise on the mathematical theory of elasticity, A.E.H. Love, University press, London, United Kingdom, 1927

[14] Theory of elasticity, S.P. Timoshenko, J.N. Goodier, Mc Graw Hill ,New York, USA, 1970

[15] The phenomenon of rupture and flow in solids, A.A. Griffith, pp. 163-198, Vol. 221, Philosophical Transactions of the Royal Society of London, United Kingdom, 1921

[16] A contribution to the theory of elasticity of non-isotropic materials (with applications to problems of bending and torsion), E. Reissner, pp. 418-428, Vol. 30, Philosophical Magazine, 1940

[17] Three-point bending of transversely isotropic rock-type materials: an analytical, numerical and experimental study, G.E. Exadaktylos, S.K. Kourkoulis, 4th Greek Association of Computational Mechanics - Congress on Computational Mechanics, Patras, Greece, 27-29 June 2002

[18] Local strains due to punch effect in three-point bending of marble beams, S.K. Kourkoulis, M.C. Stavropoulou, I. Vardoulakis, G.E. Exadaktylos, pp. 623-626, 9th Internation Congress on Rock Mechanics, Paris, France, 1999. 